\title{
The bZIP transcription factor FpAda1 is essential for fungal growth and conidiation in Fusarium pseudograminearum
}

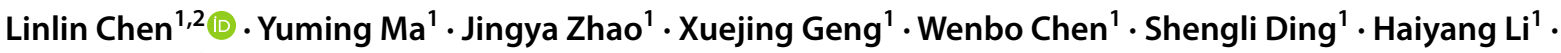 \\ Honglian $\mathrm{Li}^{1,2}$
}

Received: 15 September 2019 / Revised: 22 October 2019 / Accepted: 25 October 2019 / Published online: 6 November 2019

(c) The Author(s) 2019

\begin{abstract}
Fusarium pseudograminearum is an important pathogen of Fusarium crown rot and Fusarium head blight, which is able to infect wheat and barley worldwide, causing great economic losses. Transcription factors (TFs) of the basic leucine zipper (bZIP) protein family control important processes in all eukaryotes. In this study, we identified a gene, designated FpAdal, encoding a bZIP TF in F. pseudograminearum. The homolog of FpAda1 is also known to affect hyphal growth in Neurospora crassa. Deletion of FpAdal in F. pseudograminearum resulted in defects in hyphal growth, mycelial branching and conidia formation. Pathogenicity assays showed that virulence of the $\Delta$ fpadal mutant was dramatically decreased on wheat coleoptiles and barley leaves. However, wheat coleoptile inoculation assay showed that $\Delta$ fpadal could penetrate and proliferate in wheat cells. Moreover, the FpAda1 was required for abnormal nuclear morphology in conidia and transcription of $F p C d c 2$ and $F p C d c 42$. Taken together, these results indicate that FpAda1 is an important transcription factor involved in growth and development in F. pseudograminearum.
\end{abstract}

Keywords Fusarium pseudograminearum · Transcription factors · FpAda1 · Fungal growth · Cell cycle

\section{Introduction}

The plant pathogen Fusarium pseudograminearum is the causative agent of Fusarium crown rot (FCR) in wheat and barley, resulting in substantial yield losses worldwide (Kazan and Gardiner 2018). Particularly, in the Huanghuai wheat-growing region of China, it has been reported that $F$. pseudograminearum was the dominant pathogen of FCR (Li et al. 2012; Zhou et al. 2019). F. pseudograminearum

Communicated by M. Kupiec.

Electronic supplementary material The online version of this article (https://doi.org/10.1007/s00294-019-01042-1) contains supplementary material, which is available to authorized users.

Wenbo Chen

wenbochen@henau.edu.cn

Honglian $\mathrm{Li}$

honglianli@sina.com

1 College of Plant Protection, Henan Agricultural University, Zhengzhou 450000, China

2 National Key Laboratory of Wheat and Maize Crop Science, Zhengzhou 450000, China was initially recognized as a population within the Fusarium graminearum species group (group 1). However, F. pseudograminearum is heterothallic and it was segregated by molecular analyses (Aoki and O'Donnell 1999; Gardiner et al. 2018). Like $F$. graminearum, $F$. pseudograminearum also causes Fusarium head blight (FHB) and produces deoxynivalenol (DON) mycotoxin under favorable conditions (Kazan and Gardiner 2018; Obanor et al. 2013). Despite the devastating effects caused by FCR and FHB, establishing effective disease management strategies has been very difficult. Therefore, understanding the molecular mechanism of pathogenicity in F. pseudograminearum is of utmost relevance, given its value in the design of a proper strategy for FCR and FHB disease management.

Transcription factors (TFs) are DNA-binding proteins that interact with other components of the transcriptional machinery to regulate the expression of multiple genes. TFs can be classified into several categories based on primary and/or three-dimensional structure similarities in the DNAbinding and multimerization domains (Riechmann et al. 2000; Warren 2002). The family of transcription factors containing a basic leucine zipper domain (bZIP) is widely distributed across eukaryotes (Hurst 1995; Kong et al. 2015). 
In plants, bZIP proteins are the largest protein family, which regulate processes including abiotic stress, seed maturation, flower development and pathogen defense (Alves et al. 2013; Amorim et al. 2017). In Saccharomyces cerevisiae, the bZIP TF family contains 14 genes, and the largest is the YAP1 group, formed by eight members. Five YAP1 family members (YAPs 1, 2, 4, 5 and 6) have been implicated in oxidative stress and DNA-damage responses (He and Fassler 2005; Tan et al. 2008; Workman et al. 2006). Filamentous fungi typically contain well over a dozen of these TFs. Several fungal bZIPs have been characterized and implicated in multiple phenomena including remediation of development, amino acid biosynthesis, unfolded protein response, nutrient utilization and various stress responses (Guo et al. 2010; Kong et al. 2015; Son et al. 2011). The bZIP protein AP-1 is essential for pathogens' growth, development, infection and pathogenicity in Magnaporthe oryzae, Ustilago maydis and Colletotrichum gloeosporioides, among others (Guo et al. 2011; Li et al. 2017; Molina and Kahmann, 2007). In $N$. crassa, out of nine characterized bZIP members, Ada-1 (all development altered-1) is unique by regulating growth under minimal media conditions (Colot et al. 2006; Tian et al. 2011). In $F$. graminearum, a total of 22 bZIP TFs were functionally analyzed, and six TFs were associated with growth and pathogenicity. Among these, deletion of an Ada1 homolog (GzbZIP001) resulted in growth and virulence defects in F. graminearum (Son et al. 2011). However, there has been no research on Ada-1-like transcription factor in $F$. pseudograminearum, and their regulatory mechanism is not clear.

Cell cycle regulation is pivotal for proper cell division and cellular differentiation in eukaryotic cells. The central regulators that govern eukaryotic cell cycle progression are cyclin-dependent kinases and their partners (Bloom and Cross 2007; Humphrey and Pearce 2005; Sendinc et al. 2015). In model organisms such as yeast and $N$. crassa, Cdc2 is essential for cell cycle progression and hyphal growth (Booher and Beach 1986; Borkovich et al. 2004). F. graminearum has two $C d c 2$ genes, $C d c 2 A$ and $C d c 2 B$. The two Cdc2 orthologs have reproduction functions in hyphal growth and asexual reproduction, and only $\mathrm{Cdc} 2 \mathrm{~A}$ is important for plant infection and sexual reproduction (Jiang et al. 2016; Liu et al. 2015). Cdc42p is a Rho family GTPase, required for changes in polarized growth during mating and pseudohyphal development in S. cerevisiae. Cdc42p homologs in higher organisms are also associated with changes in cell shape and polarity (Moran et al. 2019; Rincon et al. 2014). The Cdc42 homolog has also been found in many fungi strains, and is required for hyphal growth (Bassilana et al. 2005; Boyce et al. 2001).

In this study, we identified FpAda1 as a homolog of the bZIP transcription factor Ada-1 in F. pseudograminearum, which was found to be involved in hyphal growth, conidiation and pathogenicity. Nuclear formation and the expression of cyclin-dependent protein kinase genes in $\Delta$ fpadal was also studied to understand its possible regulatory network.

\section{Materials and methods}

\section{Sequence analysis of FpAda1}

The Ada-1 (all development altered-1) gene (locus NCU00499) of N. crassa was downloaded from NCBI and used as the query to search against the $F$. pseudograminearum genome by BlastP and tBlastN algorithms (Altschul et al. 1990; Gardiner et al. 2018). The b-ZIP domain of FpAda1 was predicted by SMART (http://smart.emblheidel berg.de).

\section{qRT-PCR analyses}

For total RNA extraction, conidia were induced in CMC medium at $150 \mathrm{rpm}, 25^{\circ} \mathrm{C}$ in the dark for 4 days. Mycelia were obtained by cultivating conidia with YEPD liquid medium at $25^{\circ} \mathrm{C}, 150 \mathrm{rpm}$ for $12 \mathrm{~h}$ and were then harvested by filtration over two layers of miracloth and washed with sterilized water. For conidial infection (IF18 h to IF7 days), wheat cultivar Aikang 58, which is susceptible to F. pseudograminearum, was grown in a greenhouse at $25^{\circ} \mathrm{C}$ for 4 days. Two milliliters of conidia suspension $\left(1 \times 10^{7} / \mathrm{ml}\right)$ was infected on each coleoptile of wheat seedlings. After $18 \mathrm{~h}, 30 \mathrm{~h}, 2$ days, 3 days, 5 days and 7 days' incubation in dark at $25{ }^{\circ} \mathrm{C}$, lesion areas with $5 \mathrm{~mm}$ extension were harvested. Total RNA was extracted from each sample with the RNAsimple Total RNA Kit (Tiangen, China) according to the manufacturer's protocol. RNA was further purified and cDNA was synthesized using PrimeScript ${ }^{\mathrm{TM}} \mathrm{RT}$ reagent Kit with gDNA Eraser (Takara, China).

The expression levels of FpAdal and tested $F p C d c 2$, $F p C d c 25, F p C d c 42$ and $F p B u b 1$ were determined by quantitative real-time PCR (qRT-PCR) using the primers listed in Supplementary Table S1. For each sample, the FpTEF1 gene was used as an internal control, and the following conditions were used for the qRT-PCR reaction: $95{ }^{\circ} \mathrm{C}$ for $30 \mathrm{~s}, 40$ cycles at $95{ }^{\circ} \mathrm{C}$ for $5 \mathrm{~s}$ and $60{ }^{\circ} \mathrm{C}$ for $31 \mathrm{~s}$ to calculate cycle threshold values, followed by a dissociation program of $95{ }^{\circ} \mathrm{C}$ for $15 \mathrm{~s}, 60^{\circ} \mathrm{C}$ for $1 \mathrm{~min}$, and $95{ }^{\circ} \mathrm{C}$ for $15 \mathrm{~s}$ to obtain melt curves. The transcript levels of test genes were determined according to the function $\Delta C_{\mathrm{T}}=C_{\mathrm{T}}$ (test gene) $-C_{\mathrm{T}}$ (reference gene). To compare untreated and treated expression levels, the function $\Delta \Delta C_{\mathrm{T}}$ was determined using the equation $\Delta \Delta C_{\mathrm{T}}=\Delta C_{\mathrm{T}}$ (treatment) $-\Delta C_{\mathrm{T}}$ (control) where the control was mock-treated with $F$. pseudograminearum mycelia. The induction ratio of treatment/control was then calculated $2^{-\triangle \Delta C T}$. 


\section{Generation of the FpAda1 deletion mutant and complementation strains}

The split-marker approach was used to generate genereplacement constructs for the FpAdal gene as described in our previous study (Chen et al. 2019a). Primers are listed in Supplementary Table $\mathrm{S} 1$ and a schematic diagram of primers located for gene replacement with split-marker strategy and screening of mutant is shown in Fig. 2a. Briefly, the 1147-bp upstream and 1125-bp downstream flanking sequences were amplified with primer pairs F1/R1 and F2/R2, respectively. The hygromycin gene ( $h p h)$ was amplified from pkov 21 with primer pairs HYG/F and HYG/R. After three PCR cycles, a 1911-bp fusion PCR product including 5'-flanking region and 5'-hph region was obtained by overlap PCR amplification with primer pair $\mathrm{A} 1+\mathrm{HY} / \mathrm{R}$ using mixed fragments of FpAdal upstream and $h p h$ fragments as templates. At the same time, a 2188-bp fusion PCR product including 3'-hph region and 3'-flanking region was obtained by overlap PCR amplification with primer pair $\mathrm{YG} / \mathrm{F}+\mathrm{B} 2$ using mixed fragments of FpAdal downstream and $h p h$ fragments as templates. Products obtained by the third PCR cycle were used for fungal transformation. Putative gene deletion mutants were identified by PCR assays using the primers G1/G2, H2F/H2R, F3/H1 R and H1F/R3. Genome DNA was digested by $E c o R$ I and separated by agarose gel electrophoresis. The hygromycin gene was detected by the DIG DNA Labeling and Detection Kit (Roche, USA) according to the manufacturer's protocol.

The plasmid pYIP-102 was used for construction of the complementation vector. The FpAdal gene with its native promoter was amplified using primers ComF/ComR and inserted into the vector. The constructed vector was transformed into a $\Delta$ fpadal mutant. The complemented transformants were confirmed by western blot analysis.

\section{Phenotype determination}

For mycelial growth assays, 5-mm mycelial plugs were taken from the edge of a 3-day-old colony of each strain and placed on PDA plates and incubated at $25{ }^{\circ} \mathrm{C}$. Mycelial morphology was observed $12 \mathrm{~h}$ later, and colony diameters were measured and photographed 3 days later. For the conidiation assay, two 5-mm plugs from the edge of a 3 -day-old colony of each strain were inoculated in $100 \mathrm{ml}$ CMC. After 4 days' cultivation in a 150-rpm-shaker at $25^{\circ} \mathrm{C}$, conidia were harvested by filtering through a layer of miracloth and counted using a hemocytometer. For the conidia germination assay, $0.1 \mathrm{ml}$ of $10^{4}$ conidia/ml suspension was prepared and cultured in sterile distilled water at $25^{\circ} \mathrm{C}$ in the dark for $3 \mathrm{~h}$ and $6 \mathrm{~h}$. Three biological replicates were used for each strain and each experiment was repeated three times independently. Data were analyzed using a Student's $t$ test. To probe for nuclei, $2 \mu \mathrm{M}$ DAPI dilactate (Takara, China) was used.

\section{Pathogenicity assays}

For virulence on wheat coleoptiles, 5-mm mycelial plugs from the edge of a 3-day-old PDA plate of each strain were inoculated onto the wheat coleoptiles of the susceptible cultivar Aikang 58. The fungal discs were removed after $24 \mathrm{~h}$, and seedling lesion lengths were photographed at 3 days post-inoculation (dpi). All experiments were performed three times with five replicates per experiment. For virulence on malting barley leaves, barley seeds were planted in pots for 14 days, and then 5-mm mycelial plugs from the edge of a 3-day-old PDA plate of each strain were inoculated onto the barley leaves. All experiments were performed three times with five replicates per experiment. For pot-culture experiments, susceptible wheat cultivar Aikang 58 plants were planted in sterile soil mixing $0.5 \%$ inoculation millet for 10 days. Then wheat growth and infection were analyzed and documented. For observation of mycelium growth in infected coleoptiles, the inoculated wheat coleoptiles were harvested after $30 \mathrm{~h}$, and epidermal cells were viewed under a Nikon Ti-s instrument.

\section{Results}

\section{Identification and expression of FpAda1 in F. pseudograminearum}

One putative all development altered-1 gene (FPSE_04421, designated as FpAdal) in $F$. pseudograminearum was retrieved by BLAST search of the $F$. pseudograminearum genome with the $N$. crassa Ada-1 (NCU00499) as a query. The FpAdal gene is predicted to encode a 598-amino acid protein showing $61 \%$ identity match to $N$. crassa Ada-1. The domain analysis showed that FpAda1 has a conserved bZIP DNA-binding domain (Fig. 1a).

To further investigate the potential functions of FpAdal gene during development and pathogenicity in F. pseudograminearum, total RNA samples of mycelia, conidia and conidial infection wheat plants (IF18 $\mathrm{h}$ to IF7 days) were obtained. By qRT-PCR we observed that FpAdal expression was induced during conidiation and early infection stages (IF18 h and IF30 h), and a high transcriptional level of FpAdal was also detected at IF5 days (Fig. 1b). These results indicate that FpAda1 might play roles in both conidiation and virulence. 

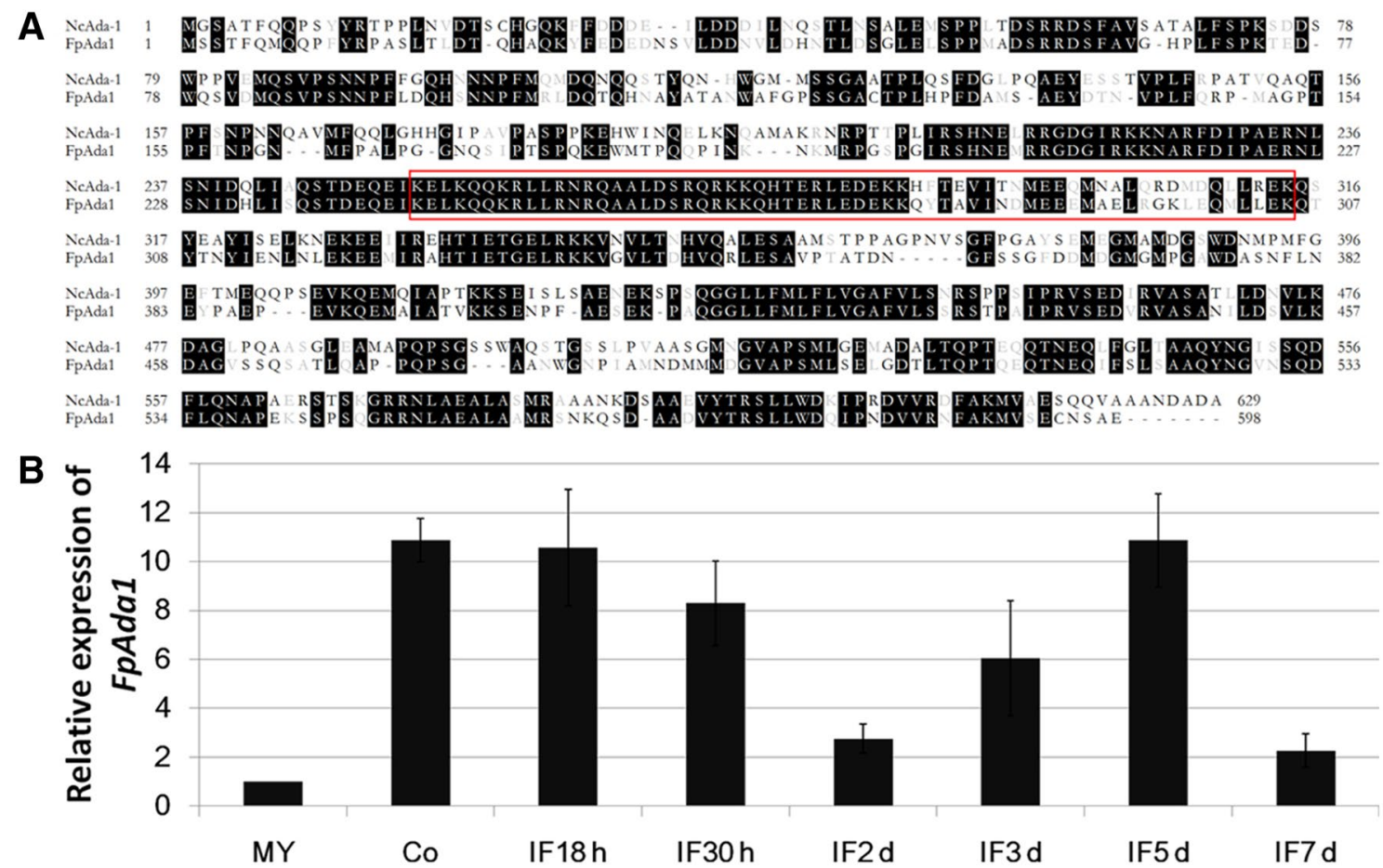

Fig. 1 Sequence alignment and expression profiles of FpAdal. a Sequence alignment of the predicted amino acid sequence of FpAda1 with its ortholog from Neurospora crassa (NcAda-1). The red box indicates the b-ZIP domain. b Expression of FpAdal in hyphae, conidia, and infected wheat coleoptiles from $18 \mathrm{~h}$ to 7 days post fertilization
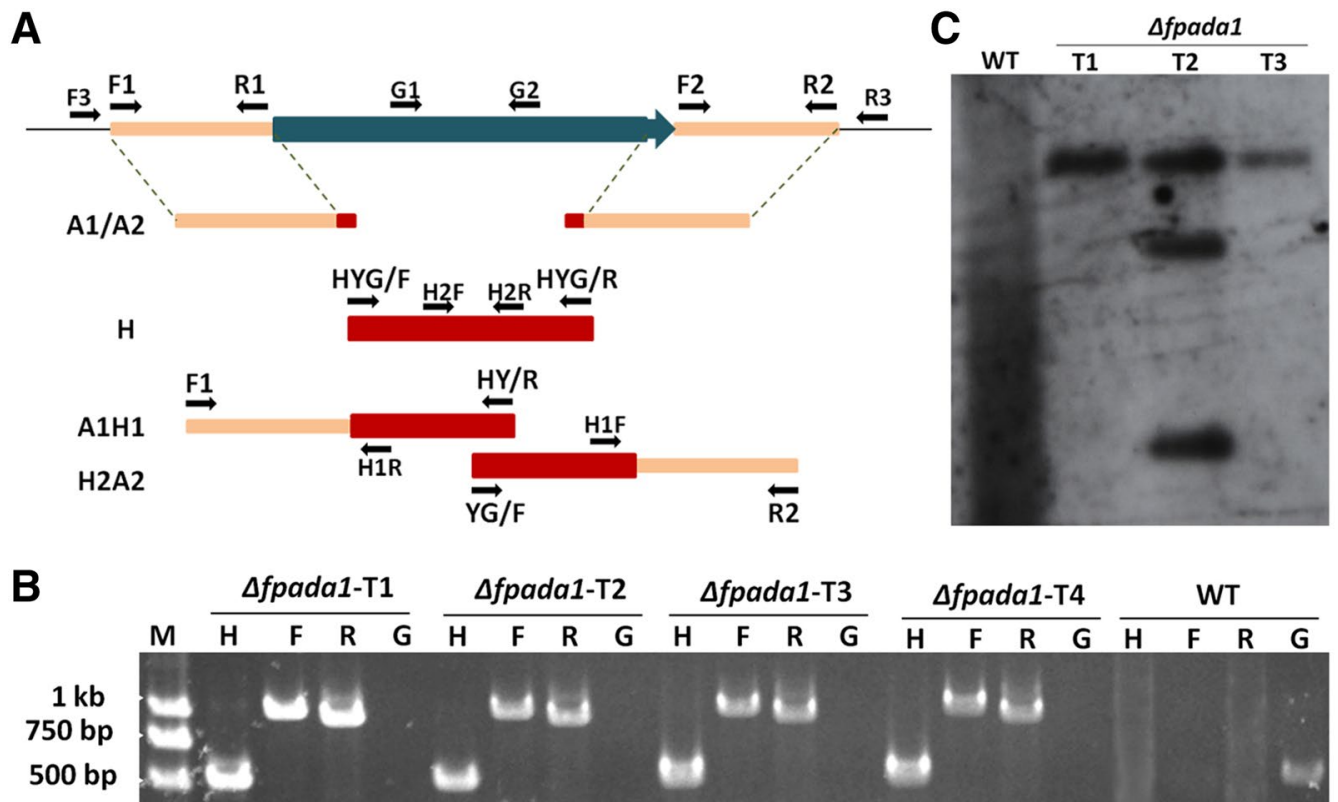

Fig. 2 Generation and identification of FpAdal gene deletion mutant. a Gene deletion strategy for FpAdal. Primers used for gene replacement and screening of mutant are indicated by arrows. b Confirmation of FpAdal deletion mutants by PCR strategy. Verification of incorporation into genomic DNA by PCR using four pairs of primers, which were used to analyze hygromycin $(\mathrm{H} 2 \mathrm{~F} / \mathrm{H} 2 \mathrm{R})$, upstream $(\mathrm{F} 3 /$ H1R), downstream (H1F/R3) and the FpAdal gene (G1/G2) positiv- ity. Amplified fragments were 523, 991, 939 and 511-bp long. WT wild-type strain WZ2-8, $M$ molecular markers, $H$ hygromycin gene, $F$ upstream, $R$ downstream, $G$ FpAdal gene. c Southern blot analysis of WT and $\Delta$ fpadal using the 750-bp DNA fragment of hygromycin as a probe. The genomic DNA preparation of each strain was digested with $E c o R$ I 


\section{Deletion and complementation of FpAda 1 gene in $F$. pseudograminearum}

To determine the biological function of FpAdal gene in $F$. pseudograminearum, FpAdal deletion mutants were generated. In Fig. 2a, a schematic diagram shows the strategy that was used to generate FpAdal gene deletion mutants and molecular verification of $\Delta$ fpadal. Transformants were selected on hygromycin-amended medium, and four individual targeted deletion mutants, designated $\Delta$ fpadal-T1, $\Delta$ fpadal-T2, 4 fpadal-T3 and $\Delta$ fpadal-T4, were created and checked by PCR (Fig. 2b). However, due to bacterial contaminations, $\Delta$ fpadal-T4 was discarded. Finally, $\Delta$ fpadal-T1 and $\Delta$ fpadal-T3 were confirmed as FpAdal gene knock-out transformants by southern blot analysis (Fig. 2c). In order to confirm that phenotypic defects in mutants were caused by FpAdal gene deletion, we complemented the mutant with a wild-type FpAdal gene with its native promoter, and a FLAG-tag was fused to the C-terminal of FpAda1. We confirmed the complemented strain $\Delta$ fpadal-C by western blot (Fig. S1).

\section{FpAda1 is critical for vegetative growth in $F$. pseudograminearum}

To evaluate the influence of FpAda1 in the vegetative growth of $F$. pseudograminearum, we examined the growth of $\Delta$ fpadal cultured on PDA medium for 3 days. Growth assessment records showed that FpAdal deletion caused a significant reduction in the strain's vegetative growth (Fig. 3a, c). In comparison with the wild-type and $\Delta$ fpadal-C strains, colony pigment deposition increased in $\Delta$ fpadal (Fig. 3a). Further microscopic examination showed that the hyphae from the $\Delta$ fpadal mutant were thinner and produced less branches at the hyphal tip as compared with the growing hyphae of the wild-type and the complemented strains (Fig. 3b). Thus, FpAda1 played an important role in the growth and hyphal branching in $F$. pseudograminearum.

\section{FpAda1 is important for conidiation in $F$. pseudograminearum}

We performed conidial production test in F. pseudograminearum to study the function of FpAda1. Cultures of the $\Delta$ fpadal mutant produced few conidia on CMC medium when compared with wild-type and $\triangle$ fpadal-C strains (Fig. 4a). After 4 days of incubation, only $5.36 \pm 0.88 \times 10^{5}$ conidia $/ \mathrm{ml}$ were obtained from the

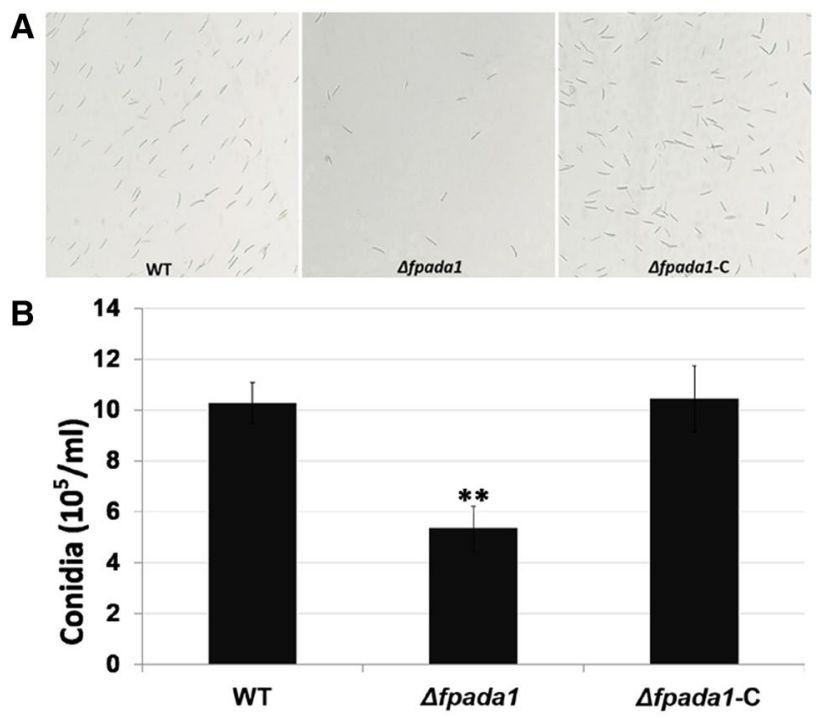

Fig. 4 Conidial production of the $\Delta$ fpadal mutant. a Conidial production (4 days after incubation in liquid CMC) of WT, $\Delta$ fpadal mutants and the complemented strain $\Delta$ fpadal-C were examined by microscopy. b Number of conidia produced by each line was measured at 4 dai. Data shown are representative of three separate experiments. The bars indicate standard error. $* * P<0.01$ ( $t$ test)
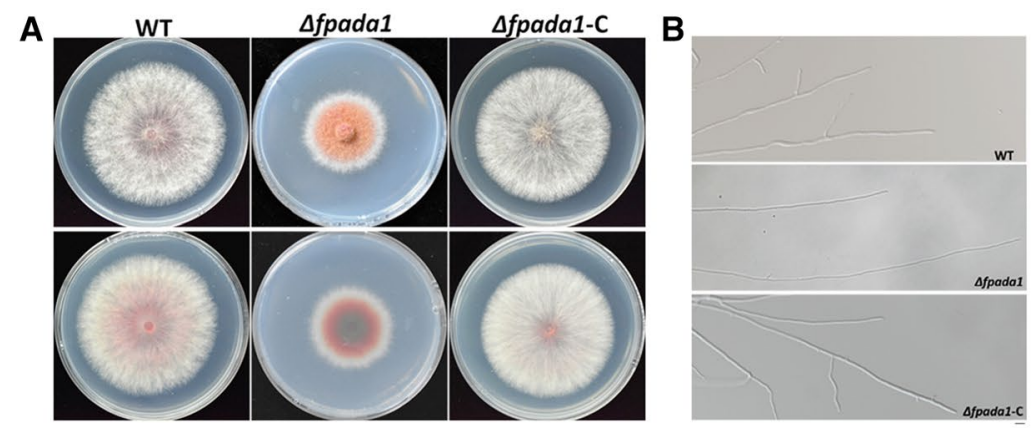

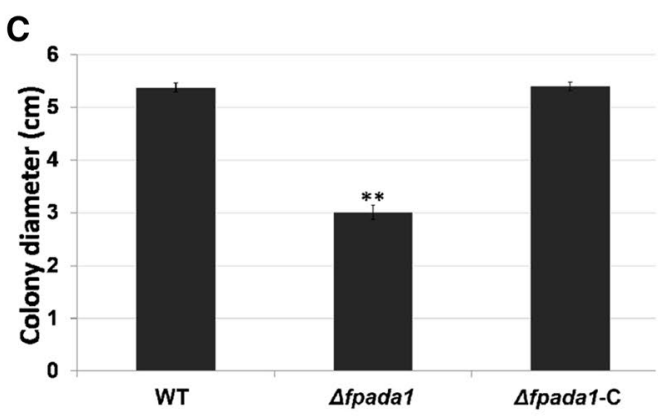

Fig. 3 Hyphal growth and pigment formation of the $\Delta$ fpadal mutant. a The WT, $\Delta$ fpadal mutants and the complemented strain $\Delta$ fpadal- $C$ were grown on PDA plates for 3 days. b Colony diameters were assayed. Linear bars in each column denote standard errors of three experiments. Two asterisks indicate significant difference of colony diameter $(P<0.01)$. $\mathbf{c}$ Hyphal tip growth and branching patterns of $F$. pseudograminearum grown on PDA medium for $12 \mathrm{~h}$. Bars $=20 \mu \mathrm{m}$ 
$\Delta$ fpadal mutant, in contrast to $10.27 \pm 0.81 \times 10^{5}$ and $10.45 \pm 1.30 \times 10^{5}$ conidia/ml in WT and $\Delta$ fpadal $-\mathrm{C}$ strains, respectively (Fig. 4a). These results indicate that FgAda1 is important for conidia production in $F$. pseudograminearum.

To further study the function of FpAda1 in the development of conidiation, we monitored conidia germination in the $\Delta$ fpadal mutant. The conidia collected from WT, $\Delta$ fpadal and $\Delta$ fpadal-C were incubated in sterile distilled water. After $3 \mathrm{~h}$, the conidia in the mutant were able to germinate and we saw a synchronization compared to the wild-type and $\Delta$ fpadal-C strains (Fig. 5a). However, the tube length of $\Delta$ fpadal was obviously shorter than that of WT and $\Delta$ fpadal-C strains after $6 \mathrm{~h}$ (Fig. $5 \mathrm{~b}$ ), which might be a consequence of a reduction in growth rate (Fig. 3a). These results indicated that FpAda1 played important roles in growth and conidiation, but not in conidia germination.
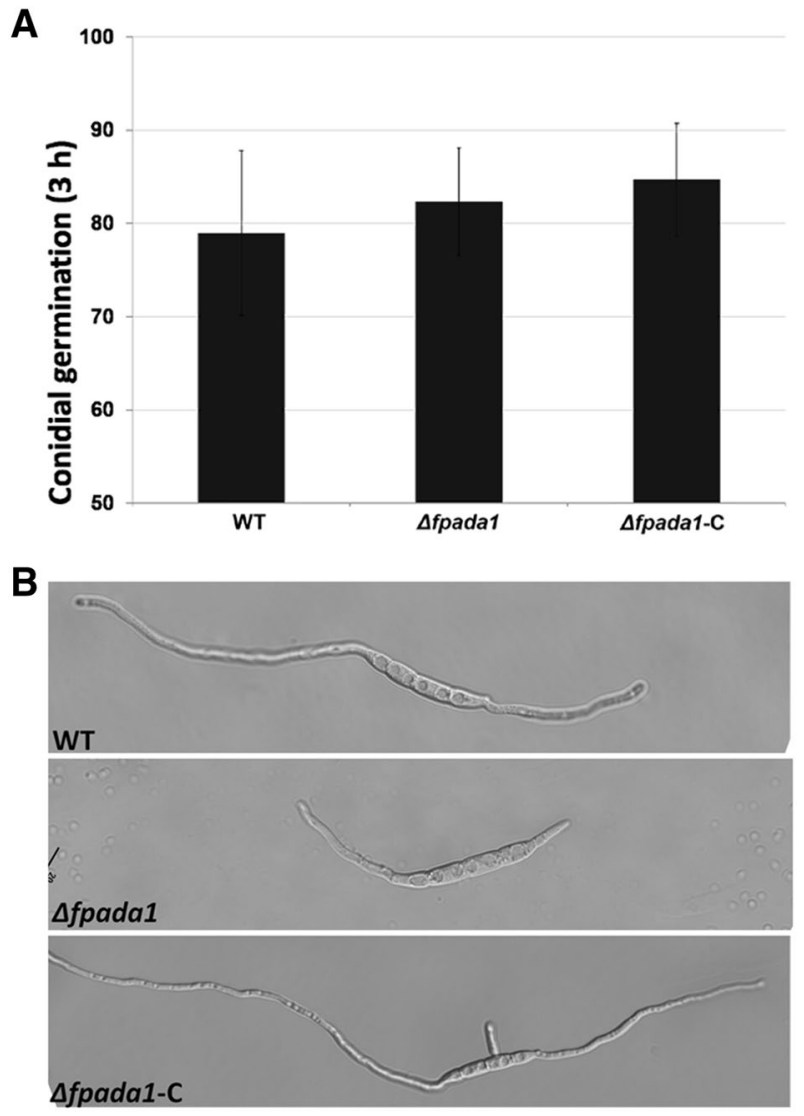

Fig. 5 Conidial germination of the $\Delta$ fpadal mutant. a Conidia germination rates were measured at $3 \mathrm{~h}$ after incubation in three independent biological replicates, each of which comprised of at least five glass slides. The bars indicate standard errors. b Conidia germlings ( $6 \mathrm{~h}$ after incubation in water) were examined by microscopy. Bars $=20 \mu \mathrm{m}$

\section{FpAda1 affects the pathogenicity in $F$. pseudograminearum}

To investigate the role of FpAda1 in fungal virulence, we first inoculated wheat coleoptiles with WT, $\Delta$ fpadal and $\Delta$ fpadal-C strains. The average length of brown lesions on the wheat coleoptiles infected with the $\Delta$ fpadal mutant was $0.62 \pm 0.12 \mathrm{~cm}$, whereas those infected with the WT and $\Delta$ fpadal-C strains showed average lesion lengths of $1.13 \pm 0.15$ and $1.06 \pm 0.16 \mathrm{~cm}$, respectively (Fig. $6 \mathrm{a}$, b). Furthermore, we also inoculated the aforementioned strains on barley leaves. The $\Delta$ fpadal mutant also caused lesions at the inoculated leaves, but the mutant-caused disease effects were less pronounced as compared with the WT and $\Delta$ fpadal-C under the same conditions (Fig. 6c). Finally, a pot-culture experiment was used to further confirm the involvement of FpAda1 in fungal virulence. The WT and $\Delta$ fpadal-C caused crown rot symptoms in wheats at 10 days post inoculation (dpi). However, wheat seedlings showed mild symptoms after inoculation with the $\Delta$ fpadal mutant (Fig. 6d).

We then evaluated the effects of $\Delta f p a d a l$ mutant on the fungal invasion process in wheat at a cellular level. After inoculation of wheat coleoptiles, microscopic analysis showed that hyphae of WT, $\Delta$ fpadal and $\Delta$ fpadal-C infected and extended similarly in coleoptile cells (Fig. 6e). The results suggest that the observed reduction in virulence might be a consequence of a reduction in growth rate of $\Delta$ fpadal mutant.

\section{FpAda1 is involved in the cell cycle of $F$. pseudograminearum}

Cell cycle regulation has been shown to be important for growth and morphological changes. Because $\Delta$ fpadal exhibited severe defects in growth and development, we assessed the expression levels of nucleus and cell division cycle genes. DAPI staining revealed that the $\Delta$ fpadal cells had abnormal nuclear morphology in conidia (Fig. 7a). In WT and $\Delta$ fpadal-C strains, one disc-shaped nucleus could be observed in every cell. However, nuclei-lacking cells were widespread in $\Delta$ fpadal conidia. In addition, the expression of three cyclin-dependent protein kinase genes ( $F p C d c 2, F p C d c 25$ and $F p C d c 42$ ), involved in fungal growth, were analyzed in WT, $\Delta$ fpadal and $\Delta$ fpadal-C strains. A serine/threonine protein kinase $(F p B u b 1)$ was chosen as contrast for the cyclin-dependent protein kinase genes. The expression levels of $F p C d c 2$ and $F p C d c 42$ were significantly reduced in $\triangle$ fpadal mutant (Fig. 7b), which further supported the role of FpAda1 in cell cycle regulation. 

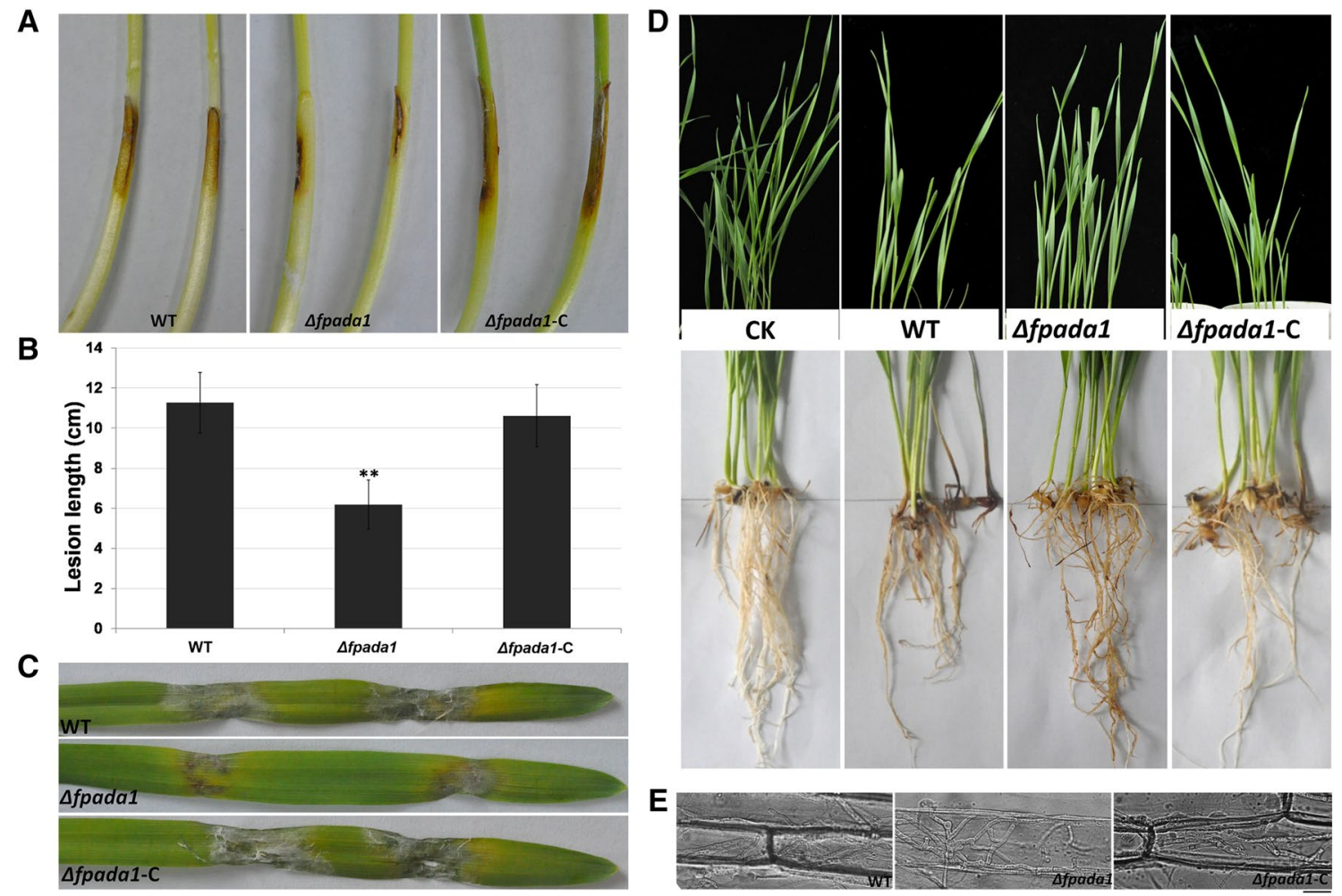

Fig. 6 Pathogenicity assays of the $\Delta$ fpadal mutant. a Wheat seedling hypocotyls were inoculated with mycelial plugs of WT, $\Delta$ fpadal mutants and the complemented strain $\Delta$ fpadal-C and examined 3 days post-inoculation. $\mathbf{b}$ Lesion lengths on wheat hypocotyls were measured at 3 days post-inoculation. The bars indicate the

standard errors. $* * P<0.01$ ( $t$ test). c Barley leaves were inoculated with mycelial plugs and examined 5 days post-inoculation. d Wheat growth and wheat root lesions were examined from the pot-culture experiment at 10 days post-inoculation. e Infection mycelia in wheat hypocotyl cells were examined at $30 \mathrm{~h}$ post-inoculation. Bars $=20 \mu \mathrm{m}$

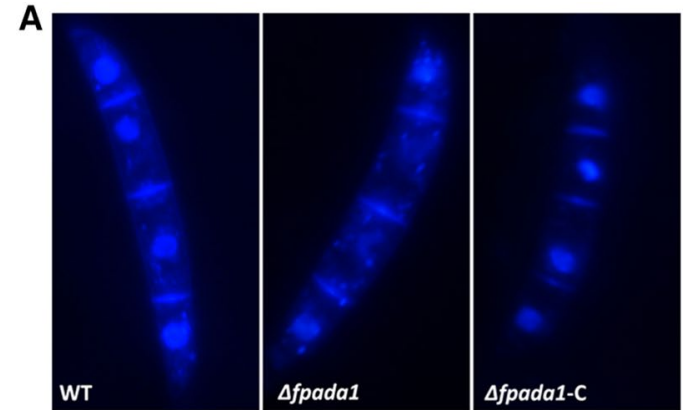

Fig. 7 a Nuclei of WT, $\Delta$ fpadal mutants and the complemented strain $\Delta$ fpadal-C were stained with DAPI and images were taken. Bars $=10 \mu \mathrm{m}$. b Relative transcription levels of cyclin-dependent protein kinase genes $(F p C d c 2, F p C d c 25, F p C d c 42)$ and serine/threonine

\section{Discussion}

The bZIP transcription factors have been reported to regulate many central physiological and developmental processes in plants, such as flowering, seed maturation, stress response and pathogen defense (Alves et al. 2013;

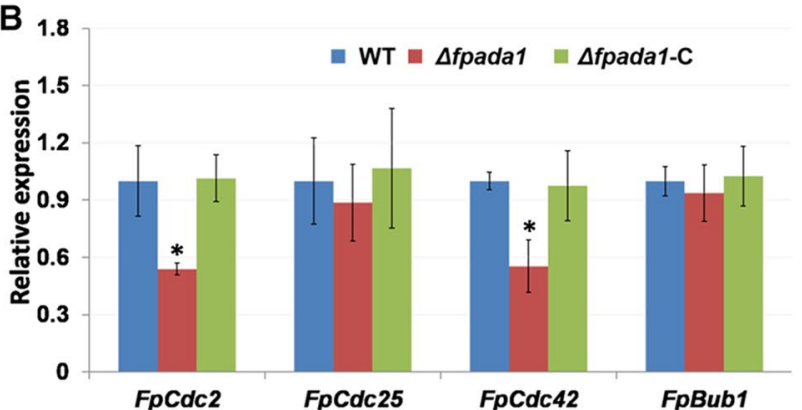

protein kinase $(F p B u b l)$ gene in WT, $\Delta f p a d a l$ mutant and the complemented strain $\Delta$ fpadal-C. For each gene, the expression level in WT was arbitrarily set as 1 . Bars denote standard errors from three repeated experiments. ${ }^{*} P<0.05$ ( $t$ test $)$

Banerjee and Roychoudhury 2017). Recently, a number of bZIP transcription factors have been identified in plant pathogenic fungi and played important roles in development, stress response and virulence. In M. oryzae, 22 bZIP transcription factors were identified and characterized as being involved in development, nutrient utilization and various stress responses (Kong et al. 2015). For example, 
MpAtf1 regulated the transcription of laccases and peroxidases, which was critical in pathogenicity (Guo et al. 2010). In $F$. graminearum, transcription factors related to growth, development, stress responses and virulence were reported (Chen et al. 2019b; Lv et al. 2019). However, few transcription factors have been described in $F$. pseudograminearum.

In this study, we have characterized a bZIP-type transcription factor FpAda1 in F. pseudograminearum as a homolog of $N$. crassa Ada-1 protein. Similar to other bZIP proteins, FpAda1 contains a bZIP DNA-binding domain. In N. crassa, the bZIP TF family contains nine genes and can be divided into two groups. Ada-1 was clustered to the GCN4 clade, and the $\Delta a d a-1$ mutant showed a reduced growth rate and very short aerial hyphae. Among the strain carrying a deletion of the $b Z I P$ gene, $\Delta a d a-1$ showed the greatest number of expression differences from the WT with 290 genes increasing, and 219 genes decreasing, consistent with its growth defect (Tian et al. 2011).

To determine the biological function of FpAda1 in $F$. pseudograminearum, its deleted mutant was generated. Compared to wild type, the mutant showed defects in hyphal growth, mycelial branching and conidia formation. However, in $F$. graminearum, the Ada- 1 homolog GzbZIP001 mutant showed no significant changes in conidiation. GzbZIP001 was required for the pathogenicity of $F$. graminearum, and its deleted mutant could not cause disease in wheat (Son et al. 2011). However, the $\Delta$ fpadal mutant could infect wheat and the deficiency in pathogenicity might be due to reduction of growth.

In fungi, cell cycle regulation has been shown to be important in terms of growth and development, and this helps ensure that cells maintain their normal size, shape and nuclear number (Ahmadian et al. 2019; Jiang et al. 2016). The cyclin-dependent protein kinases CDKs are the central regulators of the eukaryotic cell cycle (Liu et al. 2015). The $\mathrm{Cdc} 2$ kinase in yeasts and filamentous fungi has a key regulatory role in the cell cycle. Unlike other fungi, $F$. graminearum have two $C d c 2$ genes, and the two Cdc2 orthologs play different roles in vegetative and infectious hyphae (Sudbery 2008). Cdc42 is a member of the Rho family of GTPases, which are required for hyphal growth in many fungi (Nozaki et al. 2018; Si et al. 2016). Here, we found that the FpAdal deletion cells had abnormal nuclear morphology, and $F p C d c 2$ and $F p C d c 42$ were affected in expression.

In conclusion, our study demonstrated that the bZIP transcription factor plays important roles in growth, conidiation and pathogenesis in $F$. pseudograminearum. In addition, FpAda1 can affect cell cycle and the expression of $F p C d c 2$ and $F p C d c 42$.

Acknowledgements This project is supported by grants from the National Key R\&D Plan of China (2017YFD0301104), the Special
Fund for Agro-Scientific Research in the Public Interest (201503112) and the Key Research Project of Colleges and Universities in Henan Province (16A210007).

Open Access This article is distributed under the terms of the Creative Commons Attribution 4.0 International License (http://creativeco mmons.org/licenses/by/4.0/), which permits unrestricted use, distribution, and reproduction in any medium, provided you give appropriate credit to the original author(s) and the source, provide a link to the Creative Commons license, and indicate if changes were made.

\section{References}

Ahmadian M, Tyson JJ, Cao Y (2019) A stochastic model of size control in the budding yeast cell cycle. BMC Bioinform 20:322

Altschul SF, Gish W, Miller W, Myers EW, Lipman DJ (1990) Basic local alignment search tool. J Mol Biol 215:403-410

Alves MS, Dadalto SP, Goncalves AB, De Souza GB, Barros VA, Fietto LG (2013) Plant bZIP transcription factors responsive to pathogens: a review. Int J Mol Sci 14:7815-7828

Amorim LLB, da Fonseca-Dos-Santos R, Neto JPB, Guida-Santos M, Crovella S, Benko-Iseppon AM (2017) Transcription factors involved in plant resistance to pathogens. Curr Protein Pept Sci 18:335-351

Aoki T, O'Donnell K (1999) Morphological and molecular characterization of Fusarium pseudograminearum sp. nov., formerly recognized as the Group 1 population of $F$. graminearum. Mycologia 91:597

Banerjee A, Roychoudhury A (2017) Abscisic-acid-dependent basic leucine zipper (bZIP) transcription factors in plant abiotic stress. Protoplasma 254:3-16

Bassilana M, Hopkins J, Arkowitz RA (2005) Regulation of the Cdc42/ Cdc24 GTPase module during Candida albicans hyphal growth. Eukaryot Cell 4:588-603

Bloom J, Cross FR (2007) Multiple levels of cyclin specificity in cellcycle control. Nat Rev Mol Cell Biol 8:149-160

Booher R, Beach D (1986) Site-specific mutagenesis of cdc2+, a cell cycle control gene of the fission yeast Schizosaccharomyces pombe. Mol Cell Biol 6:3523-3530

Borkovich KA, Alex LA, Yarden O, Freitag M, Turner GE, Read ND, Seiler S, Bell-Pedersen D, Paietta J, Plesofsky N et al (2004) Lessons from the genome sequence of Neurospora crassa: tracing the path from genomic blueprint to multicellular organism. Microbiol Mol Biol Rev 68:1-108

Boyce KJ, Hynes MJ, Andrianopoulos A (2001) The CDC42 homolog of the dimorphic fungus Penicillium marneffei is required for correct cell polarization during growth but not development. J Bacteriol 183:3447-3457

Chen L, Geng X, Ma Y, Zhao J, Chen W, Xing X, Shi Y, Sun B, Li H (2019a) The ER lumenal Hsp70 protein FpLhs1 is important for conidiation and plant infection in Fusarium pseudograminearum. Front Microbiol 10:1401

Chen L, Tong Q, Zhang C, Ding K (2019b) The transcription factor $\mathrm{FgCrz} 1 \mathrm{~A}$ is essential for fungal development, virulence, deoxynivalenol biosynthesis and stress responses in Fusarium graminearum. Curr Genet 65:153

Colot HV, Park G, Turner GE, Ringelberg C, Crew CM, Litvinkova L, Weiss RL, Borkovich KA, Dunlap JC (2006) A high-throughput gene knockout procedure for Neurospora reveals functions for multiple transcription factors. Proc Natl Acad Sci USA 103:10352-10357 
Gardiner DM, Benfield AH, Stiller J, Stephen S, Aitken K, Liu C, Kazan K (2018) A high-resolution genetic map of the cereal crown rot pathogen Fusarium pseudograminearum provides a near-complete genome assembly. Mol Plant Pathol 19:217-226

Guo M, Guo W, Chen Y, Dong S, Zhang X, Zhang H, Song W, Wang W, Wang Q, Lv R et al (2010) The basic leucine zipper transcription factor Moatf1 mediates oxidative stress responses and is necessary for full virulence of the rice blast fungus Magnaporthe oryzae. Mol Plant-Microbe Interact MPMI 23:1053-1068

Guo M, Chen Y, Du Y, Dong Y, Guo W, Zhai S, Zhang H, Dong S, Zhang Z, Wang Y et al (2011) The bZIP transcription factor MoAP1 mediates the oxidative stress response and is critical for pathogenicity of the rice blast fungus Magnaporthe oryzae. PLoS Pathog 7:e1001302

He XJ, Fassler JS (2005) Identification of novel Yap1p and Skn7p binding sites involved in the oxidative stress response of Saccharomyces cerevisiae. Mol Microbiol 58:1454-1467

Humphrey T, Pearce A (2005) Cell cycle molecules and mechanisms of the budding and fission yeasts. Methods Mol Biol 296:3-29

Hurst HC (1995) Transcription factors 1: bZIP proteins. Protein Profile 2:101-168

Jiang C, Xu JR, Liu H (2016) Distinct cell cycle regulation during saprophytic and pathogenic growth in fungal pathogens. Curr Genet 62:185-189

Kazan K, Gardiner DM (2018) Fusarium crown rot caused by Fusarium pseudograminearum in cereal crops: recent progress and future prospects. Mol Plant Pathol 19:1547-1562

Kong S, Park SY, Lee YH (2015) Systematic characterization of the bZIP transcription factor gene family in the rice blast fungus, Magnaporthe oryzae. Environ Microbiol 17:1425-1443

Li H, Yuan H, Fu B, Xing X, Sun B, Tang W (2012) First report of Fusarium pseudograminearum causing crown rot of wheat in Henan, China. Plant Dis 96:1065

Li X, Wu Y, Liu Z, Zhang C (2017) The function and transcriptome analysis of a bZIP transcription factor CgAP1 in Colletotrichum gloeosporioides. Microbiol Res 197:39-48

Liu H, Zhang S, Ma J, Dai Y, Li C, Lyu X, Wang C, Xu JR (2015) Two Cdc2 kinase genes with distinct functions in vegetative and infectious hyphae in Fusarium graminearum. PLoS Pathog 11:e1004913

Lv W, Wu J, Xu Z, Dai H, Ma Z, Wang Z (2019) The putative histonelike transcription factor FgHltf1 is required for vegetative growth, sexual reproduction, and virulence in Fusarium graminearum. Curr Genet 65:981

Molina L, Kahmann R (2007) An Ustilago maydis gene involved in $\mathrm{H}_{2} \mathrm{O}_{2}$ detoxification is required for virulence. Plant Cell 19:2293-2309

Moran KD, Kang H, Araujo AV, Zyla TR, Saito K, Tsygankov D, Lew DJ (2019) Cell-cycle control of cell polarity in yeast. J Cell Biol 218:171-189
Nozaki S, Furuya K, Niki H (2018) The Ras1-Cdc42 pathway is involved in hyphal development of Schizosaccharomyces japonicus. FEMS Yeast Res 18:31

Obanor F, Neate S, Simpfendorfer S, Sabburg R, Wilson P, Chakraborty S (2013) Fusarium graminearum and Fusarium pseudograminearum caused the 2010 head blight epidemics in Australia. Plant Pathol 62:79-91

Riechmann JL, Heard J, Martin G, Reuber L, Jiang C, Keddie J, Adam L, Pineda O, Ratcliffe OJ, Samaha RR et al (2000) Arabidopsis transcription factors: genome-wide comparative analysis among eukaryotes. Science 290:2105-2110

Rincon SA, Estravis M, Perez P (2014) Cdc42 regulates polarized growth and cell integrity in fission yeast. Biochem Soc Trans 42:201-205

Sendinc E, Jambhekar A, Shi Y (2015) Remodeling your way out of cell cycle. Cell 162:237-238

Si H, Rittenour WR, Harris SD (2016) Roles of Aspergillus nidulans Cdc42/Rho GTPase regulators in hyphal morphogenesis and development. Mycologia 108:543-555

Son H, Seo YS, Min K, Park AR, Lee J, Jin JM, Lin Y, Cao P, Hong SY, Kim EK et al (2011) A phenome-based functional analysis of transcription factors in the cereal head blight fungus, Fusarium graminearum. PLoS Pathog 7:e1002310

Sudbery PE (2008) Regulation of polarised growth in fungi. Fungal Biol Rev 22:44-55

Tan K, Feizi H, Luo C, Fan SH, Ravasi T, Ideker TG (2008) A systems approach to delineate functions of paralogous transcription factors: role of the Yap family in the DNA damage response. Proc Natl Acad Sci USA 105:2934-2939

Tian C, Li J, Glass NL (2011) Exploring the bZIP transcription factor regulatory network in Neurospora crassa. Microbiology 157:747-759

Warren AJ (2002) Eukaryotic transcription factors. Curr Opin Struct Biol 12:107-114

Workman CT, Mak HC, McCuine S, Tagne JB, Agarwal M, Ozier O, Begley TJ, Samson LD, Ideker T (2006) A systems approach to mapping DNA damage response pathways. Science 312:1054-1059

Zhou H, He X, Wang S, Ma Q, Sun B, Ding S, Chen L, Zhang M, Li H (2019) Diversity of the Fusarium pathogens associated with crown rot in the Huanghuai wheat-growing region of China. Environ Microbiol 21:2740-2754

Publisher's Note Springer Nature remains neutral with regard to jurisdictional claims in published maps and institutional affiliations. 